\title{
Predictive Model to Differentiate Dengue Fever from Other Febrile Illnesses in Children-Application of Logistic Regression Analysis
}

\author{
Sangeeta P Sawant ${ }^{1}$, Surekha Rudraraju ${ }^{2}$, Alpa S Amin ${ }^{3}$
}

\begin{abstract}
Background: Diagnosis of dengue fever (DF) is challenging in the initial stage of illness. Early diagnosis and adequate management are important to reduce the complications associated with dengue.

Objectives: This study aims to identify the clinical and laboratory features to predict DF from other febrile illnesses (OFI).

Materials and methods: A observational analytical study was undertaken in an urban referral hospital in Mumbai, India. Eighty-seven children (up to 14 years of age) presenting with acute fever of $>24$ hours and $<7$ days without any evident or suspected focus on clinical examination were included. Clinical features and laboratory parameters at the time of presentation were used to build a predictive model for DF by multivariate logistic regression analysis. A rapid, qualitative immunochromatographic test for the detection of dengue non-structural protein-1 (NS1) antigen and IgG and IgM antibodies to dengue virus was done as a screening test for DF in all children. A serological test including the dengue IgM (MAC ELISA) test or its seroconversion after 2 weeks was considered as a diagnostic test for DF.

Results: Dengue fever was diagnosed in $51.7 \%$ of children. Myalgia was an independent predictor of DF amongst the clinical features. A model including clinical and laboratory features demonstrated myalgia, leukopenia, and raised liver aspartate transaminase (AST) to be significant predictors of early DF. This model was internally validated, had high accuracy with an area under curve (AUC) of $0.93(95 \% \mathrm{Cl} 0.88-0.98)$ with sensitivity $86.7 \%$ (95\% Cl 72.5-94.4), specificity $83.3 \%$ (95\% Cl 68.0-92.4), positive predictive value $84.78 \%$ (95\% Cl 70.51-93.16), and negative predictive value $85.4 \%$ (95\% Cl 70.13-93.9).

Conclusion: We constructed a predictive model for the diagnosis of DF in an earlier stage of presentation. Validation of this model in a larger population and different regions should be attempted.
\end{abstract}

Keywords: Dengue fever, Laboratory diagnosis, Logistic regression, Predictors, Signs/symptoms.

Pediatric Infectious Disease (2021): 10.5005/jp-journals-10081-1283

\section{INTRODUCTION}

Dengue is the world's most common mosquito-borne viral infection and a leading cause of morbidity throughout the tropics and subtropics. IIIness caused by one of the four dengue virus serotypes can range from non-specific febrile illness to classic dengue fever (DF), which may progress to severe disease like dengue hemorrhagic fever or dengue shock syndrome.

In India, it is a major public health problem, the leading cause of hospitalization and death among children. There is hyperendemicity with all four serotypes and the overall estimate of the prevalence of laboratory-confirmed dengue infection among clinically suspected patients has been reported to be $38.3 \%{ }^{2}$

Clinical features of dengue vary significantly between age groups and stage of illness. Also, several infectious and noninfectious diseases mimic DF. It is challenging to distinguish DF from other febrile illnesses (OFI) in the early febrile phase. ${ }^{3}$

Early identification of DF can help clinicians make better decisions by recognizing the patients who need to be monitored for possible dengue complications. ${ }^{4}$

A diagnosis of dengue infection is confirmed by the detection of the virus, the viral genome or non-structural protein-1 (NS1) Ag or seroconversion of IgM or lgG. Positive IgM serology by ELISA or a hemagglutinin inhibition test (HIA) antibody titer of 1,280 or higher is diagnostic criteria of probable dengue infection. ${ }^{5}$

The NS1 antigen detection by a rapid screening test or ELISA is highly sensitive and specific. However, it has low sensitivity in case
${ }^{1-3}$ Department of Paediatrics, Bhabha Atomic Research Centre Hospital, Mumbai, Maharashtra, India

Corresponding Author: Sangeeta P Sawant, Department of Paediatrics, Bhabha Atomic Research Centre Hospital, Mumbai, Maharashtra, India, Phone: +91 9321214832, e-mail: drsawantsangeeta@gmail.com

How to cite this article: Sawant SP, Rudraraju S, Amin AS. Predictive Model to Differentiate Dengue Fever from Other Febrile Illnesses in Children-Application of Logistic Regression Analysis. Pediatr Inf Dis 2021;3(1):9-14.

Source of support: Nil

Conflict of interest: None

of secondary infection due to immune complex formation by NS1 antigen with the preexisting antibodies. ${ }^{6}$

Rapid test for NS1 Ag detection and IgM determination at the primary care level is recommended for the DF diagnosis. The limitations of the rapid kit are their variable sensitivity and specificity. They are susceptible to unfavorable storage conditions (especially in remote/rural areas) and can give false results. Also cross-reactions are possible with other diseases such as scrub typhus, tuberculosis, leptospirosis, malaria etc. ${ }^{7}$

In recent years, the focus on early clinical and laboratory predictors using regression methods has increased..$^{4,8-10} \mathrm{~A}$ predictive model can help in the early diagnosis of DF and therefore allow for rapid decision-making about its management. 
We undertook this study to identify clinical and laboratory predictive parameters using the multivariable logistic regression method to differentiate DF from OFI in children.

\section{Materials and Methods}

This single-center, observational, analytical, hospital-based study was conducted in the Paediatrics Department, Bhabha Atomic Research Centre Hospital over 2 years from October 2016 to October 2018. Ethical permission was obtained from the Institutional Review Board of the same Hospital.

The study population included children up to 14 years of age admitted with a fever of $>24$ hours and $<7$ days without an evident or suspected focus on clinical examination. Children with localizing clinical features suggestive of alternate diagnosis/confirmed illness were excluded from the study.

The average prevalence of children getting admitted with acute febrile illness in the above institute is $30 \%$. The sample size was calculated with $95 \%$ confidence level and $10 \%$ precision using the formula $\left[t^{2} \times(p \times q) / c^{2} ; t\right.$ : confidence interval, $p$ : expected proportion of cases, $q:(1-p), c$ : precision] as 81 cases.

A total of 87 children admitted with acute febrile illness were included in the study.

Patients were evaluated through comprehensive clinical history and physical examination on initial presentation. Clinical data including the presence of fever \pm chills, coryza, cough, sore throat, earache, vomiting, diarrhea, abdominal pain, reduced urine output, rash, itching, headache, prostration, myalgia, arthralgia, retro-orbital pain, photophobia, back pain loss of appetite, facial flush/rash, conjunctival redness, lymphadenopathy and hemorrhagic symptoms were recorded. Vital parameters including temperature, pulse rate, respiratory rate and blood pressure (BP) were measured. Physical signs like the presence of rash, pallor, icterus, lymphadenopathy, dehydration, conjunctival congestion, pharyngeal erythema, bleeding manifestations hepatomegaly, splenomegaly, and mental status were noted. A tourniquet test was performed within 24 hours after admission.

A blood sample was collected for hemoglobin $(\mathrm{Hb})$, hematocrit (HcT), total white blood cell (WBC), differential leukocyte count, platelet count along with serum liver transaminase, i.e. aspartate transaminase and alanine transaminase (AST and ALT) levels.

A rapid, qualitative immunochromatographic test (dengue check Combo test) for the detection of dengue NS1 antigen and differential detection of IgG and IgM antibodies to dengue virus was done as a screening test for DF in all children.

Dengue IgM (MAC ELISA) antibody test was done for all children presenting with fever of $>5$ days. Those with negative IgM antibody tests were reinvestigated for seroconversion in convalescent-phase after 2 weeks.

Positive IgM (MAC ELISA) or IgM seroconversion was considered as a diagnostic test for DF. ${ }^{5}$

\section{Case Definition}

\section{Dengue Fever}

Children with acute febrile illness of $<7$ days with dengue IgM ELISA antibody test positive or IgM seroconversion on convalescent serum.

\section{Other Febrile Illnesses}

Children with fever $<7$ days without localizing signs with negative dengue IgM ELISA antibody detected in the acute specimen or a convalescent serum.

\section{Variable Definition}

Leukopenia: WBC count $\leq 5,000 / \mathrm{mm}^{3}$.

Raised HCT: HCT $>40 \%$.

Raised liver transaminase: AST: $>40 \mathrm{IU} / \mathrm{L}$ and ALT: $>25 \mathrm{IU} / \mathrm{L}$.

\section{Statistical Methods}

The demographics, clinical characteristics, and laboratory results of DF and OFI were described using the mean \pm standard deviation (SD) if the data were normally distributed or by median and range otherwise. Comparison between the two groups was performed using the Student's $t$ test for continuous variables if the data were normally distributed otherwise, the Mann-Whitney $U$ test was used. Comparison of continuous variables among more than two groups was done using ANOVA test. A Chi-square test was used for categorical data. A $p$ value $<0.05$ was considered significant.

Univariate logistic regression was conducted to identify variables significantly associated with DF and they were included in the regression model. Multivariate logistic regression analysis was performed using the hierarchical method. The first block included the clinical variables and the second block included laboratory variables.

The clinical parameters entered in block 1 were vomiting, loss of appetite, aches (including headache body ache), lethargy, myalgia, presence of erythematous rash and conjunctival redness.

Retro-orbital pain diarrhea and splenomegaly though significant were not entered in the model as the event per variable was $<10$. $^{11,12}$

Collinearity test: A collinearity test was conducted for laboratory variables. Hemoglobin with HCT and AST with ALT were highly collinear, therefore HCT and AST were chosen along with WBC count and platelet count to enter in block 2 . These variables were converted into bivariate variables and labelled as values " 0 " (less than) and "1" (more than) for WBC count $\leq$ or $>5,000 / \mathrm{mm}^{3}$ and platelet count $\leq$ or $>250,000 / \mathrm{mm}^{3}$. Hematocrit $\leq$ or $>40 \%$, and liver AST $\leq$ or $>40 \mathrm{IU} / \mathrm{L}$.

Bootstrapping with 2,000 replicates were used for internal validation. After building and validating logistic regression models, the sensitivity of the method was determined using the receiver operating characteristic (ROC) curve. The best cut-off value for probabilities was determined by the Youden index that maximizes the sum of sensitivity and specificity.

\section{Results}

Among the 87 children (56 boys, 31 girls) presenting with fever, $51.7 \%(n=45)$ were DF and $48.3 \%$ were OFI $(n=42)$. Demographic features of DF and OFI are presented in Table 1. The median age was significantly more in the DF group than in OFI (9.0 vs 5.5 years; $p<$ $0.05)$. The youngest patient in the DF group was an 18-month-old child. Intragroup age analysis demonstrated DF to be significant in children $>10$ years compared to $<5$ years (group III $v$ group I; $p$ $<0.05$ ). Dengue fever had a greater percentage of boys than girls with a male to female ratio of 1.6:1. The history of a family member suffering from dengue was obtained in $26.6 \%$ DF cases compared to $2.3 \%$ with OFI $(p<0.001)$.

Tables $2 \mathrm{~A}$ and $2 \mathrm{~B}$ present the main presenting signs/symptoms and results of laboratory investigations. The median duration of fever was 4 days in both groups. Chills were present in $60 \%$ of DF compared to $40.5 \%$ in OFI though not statistically significant. There was no difference in respiratory symptoms like cough, cold in both groups. 
Table 1: Demographic features of dengue fever (DF) and other febrile illness (OFI)

\begin{tabular}{lllll}
\hline Demographic characteristics & $D F(N=45)$ & OFI $(N=42)$ & Total $(N=87)$ & p value \\
\hline $\begin{array}{l}\text { Age in years (median) } \\
\text { Age group }\end{array}$ & $9(5.95)$ & $5.5(6.26)$ & $7.05(6.01)$ & $0.01^{*}$ \\
$\quad<5$ years (I) & $7(31.8)$ & $15(68.2)$ & $22(25.28)$ & $0.036^{\$ *}$ \\
$\quad$-10 years (II) & $21(52.5)$ & $19(47.5)$ & $40(45.97)$ & \\
$\quad>10$ years (III) & $17(68)$ & $8(32)$ & $25(28.73)$ & \\
Gender & & & & \\
$\quad$ Boys & $26(60.5)$ & $28(68.3)$ & $56(64.4)$ & 0.785 \\
$\quad$ Girls & $17(39.5)$ & $13(31.7)$ & $31(35.6)$ & 0.273 \\
Family H/o DF & $12(26.6)$ & $1(2.3)$ & $13(14.94)$ & $0.001^{*}$ \\
\hline
\end{tabular}

${ }^{*} p$ value $<0.05$

${ }^{\$}$ ANOVA between group (I) and (III)

Table 2A: Clinical features and laboratory investigations of dengue fever (DF) and other febrile illness (OFI)

\begin{tabular}{|c|c|c|c|c|c|}
\hline Clinical and laboratory investigations & $D F(N=45)$ number $(\%)$ & OFI $(N=42)$ number (\%) & Total number (\%) & $p$ value & Odds ratio \\
\hline \multicolumn{6}{|l|}{ Symptoms } \\
\hline Fever duration (days) (median, IQR) & $4(2)$ & $4(3)$ & $4(2)$ & 0.55 & \\
\hline Chills & $27(60)$ & $17(40.5)$ & $44(50.6)$ & 0.06 & \\
\hline Cough & $27(60)$ & $21(50)$ & $37(42.5)$ & 0.173 & \\
\hline Cold & $14(31.1)$ & $19(45.2)$ & $33(37.9)$ & 0.175 & \\
\hline Vomiting & $29(64.4)$ & $17(40.5)$ & $46(52.9)$ & $0.025^{*}$ & 5.009 \\
\hline Diarrhea & 0 & $5(11.9)$ & $6(6.9)$ & $0.018^{*}$ & 5.684 \\
\hline Loss of appetite & $23(51.1)$ & $12(28.6)$ & $35(40.2)$ & $0.032^{*}$ & 4.59 \\
\hline Abdominal pain & $14(31.1)$ & $9(21.4)$ & $23(26.4)$ & 0.306 & \\
\hline Reduced urine output & $5(11.11)$ & $4(9.52)$ & $9(10.34)$ & 0.8 & \\
\hline Rash & $12(26.7)$ & $7(16.7)$ & $19(21.8)$ & 0.259 & \\
\hline Itching & $7(15.6)$ & $4(9.5)$ & $11(12.6)$ & 0.398 & \\
\hline Headache & $22(48.9)$ & $10(23.8)$ & $32(36.8)$ & $0.015^{*}$ & 5.876 \\
\hline Prostration/lethargy & $15(33.3)$ & $3(7.1)$ & $18(20.7)$ & $0.003^{*}$ & 9.081 \\
\hline Myalgia & $14(31.1)$ & $1(2.4)$ & $15(17.4)$ & $0.000^{*}$ & 13.867 \\
\hline Arthralgia & $3(6.7)$ & $1(2.4)$ & $4(4.6)$ & 0.34 & \\
\hline Backpain & $17(37.8)$ & $2(4.8)$ & $19(21.8)$ & $0.000^{*}$ & 13.873 \\
\hline Retro-orbital pain & $6(13.3)$ & 0 & $6(6.9)$ & $0.014^{*}$ & 6.015 \\
\hline \multicolumn{6}{|l|}{ Signs } \\
\hline Conjunctival redness & $14(31.1)$ & $3(7.1)$ & $17(19.5)$ & $0.005^{*}$ & 7.938 \\
\hline Lymphadenopathy & $3(6.7)$ & $8(19)$ & $11(12.6)$ & $0.08^{*}$ & 3.015 \\
\hline Erythematous rash & $21(46.7)$ & $10(23.8)$ & $31(35.6)$ & $0.026^{*}$ & 4.949 \\
\hline Pallor & $23(51.1)$ & $18(42.9)$ & $41(47.1)$ & 0.441 & \\
\hline Pharyngeal erythema & $27(60)$ & $24(57.1)$ & $51(58.6)$ & 0.787 & \\
\hline Hepatomegaly & $11(24.4)$ & $10(23.8)$ & $21(24.1)$ & 0.945 & \\
\hline Splenomegaly & $1(2.2)$ & $7(16.7)$ & $8(9.2)$ & $0.02 *$ & 5.428 \\
\hline \multicolumn{6}{|l|}{ Laboratory investigations } \\
\hline Hematocrit $>40 \%$ & $12(26.7)$ & $3(7.1)$ & $15(17.2)$ & $0.016^{*}$ & 5.803 \\
\hline WBC count $<5,000 /$ cumm & $30(66.7)$ & $7(16.7)$ & $37(42.5)$ & $0.000^{* *}$ & 22.222 \\
\hline Platetet count $<250,000 /$ cumm & $37(82.2)$ & $22(52.4)$ & $59(67.8)$ & $0.003^{* *}$ & 8.864 \\
\hline AST $>40 \mathrm{IU} / \mathrm{L}$ & $41(91.1)$ & $24(57.1)$ & $65(74.7)$ & $0.000^{* *}$ & 13.268 \\
\hline $\mathrm{ALT}>25 \mathrm{IU} / \mathrm{L}$ & $27(60)$ & $15(35.7)$ & $42(48.3)$ & $0.023^{*}$ & 5.131 \\
\hline NS1 antigen & $40(88.9)$ & $4(9.5)$ & $44(50.6 \%)$ & $0.000^{* *}$ & 54.742 \\
\hline
\end{tabular}

${ }^{*} p$ value $<0.05,{ }^{* *} p$ value $<0.005$

AST, aspartate transaminase; ALT, alanine transaminase

Children with DF had significantly increased frequency of vomiting (64.4\%), loss of appetite (51.1\%), headache $(48.9 \%)$, backache (37.8\%), prostration/lethargy (33.3\%), myalgia
(31.1\%) and retro-orbital pain (13.3\%). Diarrhea was significant in OFI (11.9\%) while no patient in the DF group had this symptom. 
Predictive Model to Differentiate Dengue Fever from Other Febrile Illnesses in Children

Table 2B: Clinical features and laboratory investigations of dengue fever (DF) and other febrile illness (OFI)

\begin{tabular}{|c|c|c|c|c|}
\hline Clinical and laboratory investigations & $D F(N=45)$ & OFI $(N=42)$ & All cases & pvalue \\
\hline \multicolumn{5}{|l|}{ Signs } \\
\hline Pulse rate (median, IQR) & $107.26(13.911)$ & $112.2(1.273)$ & $110(22)$ & 0.143 \\
\hline Resp rate (median, IQR) & $24.14(3.889)$ & $24.93(5.101)$ & $24(4)$ & 0.618 \\
\hline SBP mm Hg (median, IQR) & $103.74(10.461)$ & $96.37(10.119)$ & $100(12)$ & $0.004^{* *}$ \\
\hline DBP mm Hg (median, IQR) & $65.49(8.282)$ & $61.1(8.264)$ & $60(10)$ & $0.026^{*}$ \\
\hline \multicolumn{5}{|l|}{ Laboratory investigations } \\
\hline $\mathrm{Hb}$ g\% (mean $\pm \mathrm{SD})$ & $12.61(1.07)$ & $11.87(1.27)$ & $12.25(1.22)$ & $0.005^{* *}$ \\
\hline $\mathrm{HcT}($ mean $\pm \mathrm{SD})$ & $38.01(3.076)$ & $35.58(2.92)$ & $36.82(3.22)$ & $0.000^{* *}$ \\
\hline WBC count $/ \mathrm{mm}^{3}$ (median, IQR) & $4,280(2,788)$ & $7,900(4,195)$ & $5,400(4,890)$ & $0.000^{* *}$ \\
\hline Neutrophil \% (mean \pm SD) & $50.69(17.42)$ & $56.85(14.09)$ & $53.50(16.09)$ & 0.76 \\
\hline Lymphocyte \% (median, IQR) & $39.9(27.2)$ & $29(18.6)$ & $36(24.7)$ & 0.112 \\
\hline Platelet count/cumm (mean \pm SD) & $200,295(58,179)$ & $244,111(94,360)$ & $222,055(80,984)$ & $0.01 *$ \\
\hline AST (IU/L) (median, IQR) & $63.5(52)$ & $43(30)$ & $53(35)$ & $0.000^{*}$ \\
\hline ALT (IU/L) (median, IQR) & $28(26)$ & $20(17)$ & $25(19)$ & $0.015^{*}$ \\
\hline Blood urea nitrogen (mean $\pm S D)$ & $9.1(2.91)$ & $10.06(3.68)$ & $9.56(3.32)$ & 0.18 \\
\hline Serum creatinine (median, IQR) & $0.6(0.2)$ & $0.5(0.1)$ & $0.5(0.1)$ & $0.023^{*}$ \\
\hline
\end{tabular}

${ }^{*} p$ value $<0.05 ;{ }^{* *} p$ value $<0.005$

Resp rate: respiratory rate/minute; SBP, systolic blood pressure; DBP, diastolic blood pressure. Hb, hemoglobin; HcT, hematocrit; WBC, white blood cell; AST, aspartate transaminase; ALT, alanine transaminase

Table 3: Adjusted odds ratio and accuracy parameters of the multiple logistic regression model (clinical parameters only AND clinical and laboratory parameters) for diagnosis of dengue fever

\begin{tabular}{|c|c|c|c|c|c|c|}
\hline \multirow{2}{*}{$\begin{array}{l}\text { Logistic regression } \\
\text { model }\end{array}$} & \multicolumn{2}{|c|}{ Adjusted odds ratio } & \multicolumn{2}{|c|}{ Sensitivity } & \multicolumn{2}{|c|}{ Specificity } \\
\hline & OR & $95 \% \mathrm{Cl}$ & $\%$ & $95 \% \mathrm{Cl}$ & $\%$ & $95 \% \mathrm{Cl}$ \\
\hline \multicolumn{7}{|c|}{ Model 1-clinical parameters only } \\
\hline Myalgia & 17.642 & $1.501-22.105$ & 73.3 & $57.8-84.9$ & 81 & $65.3-90.8$ \\
\hline \multicolumn{7}{|c|}{ Model 2-clinical and laboratory parameters } \\
\hline Myalgia & 13.46 & $0.71-78.175$ & 86.7 & $72.5-94.4$ & 83.3 & $68.0-92.4$ \\
\hline WBC $<5,000 /$ cumm & 16.325 & $1.003-86.927$ & & & & \\
\hline AST $>40 \mathrm{IU} / \mathrm{L}$ & 11.118 & $0.058-82.693$ & & & & \\
\hline
\end{tabular}

OR, odds ratio; $95 \% \mathrm{Cl}, 95 \%$ confidence interval; WBC, white blood cell count; AST, aspartate transaminase

On physical examination, presence of erythematous rash $(46.7 \%)$ and conjunctival redness (31.1\%) were significant signs in DF and splenomegaly was significant in OFI. There was no significant difference between the vital parameters except the median systolic BP (SBP) and diastolic BP (DBP) which were significantly different $(p<0.05 \%)$ in the DF group. None of the children presented with hemorrhagic manifestations at the time of presentation.

In the DF group, laboratory results demonstrated significantly low median WBC, platelet count, and higher mean Hb, HcT, AST, and ALT.

The NS1 antigen test was positive in $88.8 \%$ of DF. It had a sensitivity of $88.8 \%$ and specificity of $90.5 \%$.

Univariate analysis was performed for clinical and laboratory variables. Multivariate regression analysis results demonstrated that after the addition of clinical features in block 1, the predictive value of the model increased significantly from 51.7 to $77 \%$ ( $p<$ 0.001). Myalgia was observed to be a significant clinical predictor of DF. Block 2 consisted of laboratory parameters. The predictive value now increased to $85.1 \%(p<0.001)$. In the final model, myalgia, WBC count $<5,000 / \mathrm{mm}^{3}$, and AST $>40$ IU were the independent predictors for DF.
Bootstrapping with 2,000 replicates was performed for internal validation. The results of multiple regression analyses displaying adjusted odds ratios for each model variable are shown in Table 3.

The logistic regression model had a good overall prediction and this was confirmed through the receiver operating characteristic (ROC) curve analysis. Group probabilities were calculated using the equation and the ROC curve was plotted (Fig. 1). It had excellent accuracy with an area under curve (AUC) of $0.93(\mathrm{Cl} 0.88-0.98)$ and a standard error of 0.026 . At 0.5 cut-off value, this model showed sensitivity $86.7 \%(95 \% \mathrm{Cl} 72.5-94.4)$, specificity $83.3 \%(95 \% \mathrm{Cl}$ 68.0-92.4), positive predictive value $84.78 \%$ (95\% Cl 70.51-93.16) and negative predictive value $85.4 \%$ (95\% Cl 70.13-93.9).

\section{Discussion}

Early diagnosis of dengue may be challenging as the presentation is with non-specific signs and symptoms which may not be easily differentiated from OFI.

We observed a high median age and male predominance in DF. The higher age of children with DF has been reported in most of the published studies. ${ }^{13-15}$ However, a population-based study from north and south India observed DF in younger children. ${ }^{16,17}$ 


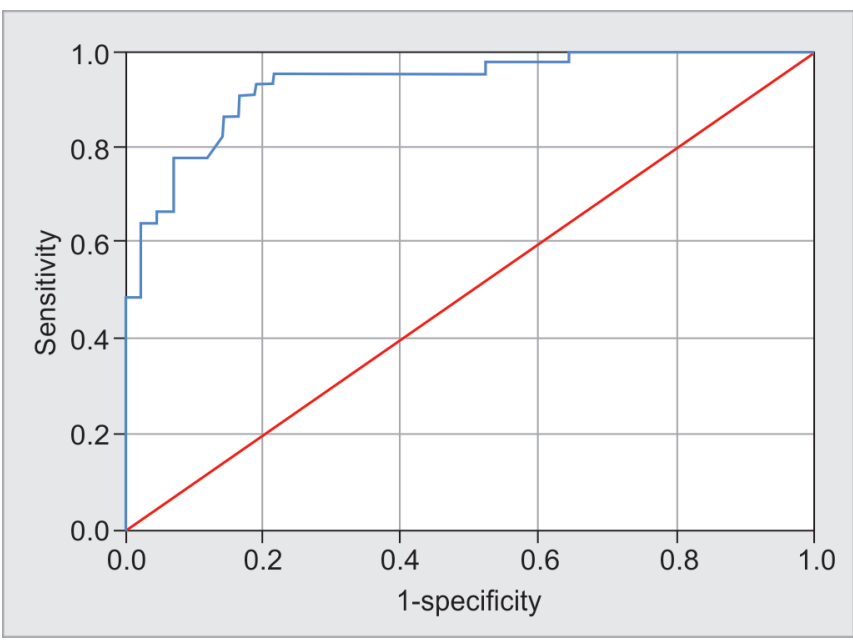

Fig. 1: Receiver-operating characteristic (ROC) curve for the logistic regression model with clinical and laboratory data

The demographic features observed in the present study were similar to the studies conducted during the same time period in other parts of the country. ${ }^{13,14,16}$

We identified certain clinical and laboratory features associated with DF. Fever associated with nausea/vomiting, loss of appetite headache, body ache, lethargy, myalgia, retro-orbital pain, presence of erythematous rash, conjunctival redness, and high $\mathrm{Hb}, \mathrm{HcT}$ low WBC and platelet count along with raised AST, ALT were the significant clinical and laboratory features associated in the early stages of DF. Children with DF had significantly high BP readings which could be explained due to the higher mean age of children with DF.

The clinical features like retro-orbital pain, absence of diarrhea, and splenomegaly were exclusive features of DF in our study. It is important to highlight here that the presence of a rash on physical examination was accurate for DF diagnosis than its history as a symptom. This observation was similar to those reported in the published literature. ${ }^{10,18-20}$

Fever with a combination of various clinical features and laboratory parameters along with its association with DF has been reported..$^{10,13-15,20-23}$

It is unlikely that any one indicator is useful in clinical practice because these signs and symptoms are present in other diseases too; hence, a combination of clinical features and laboratory parameters may help to distinguish DF from OFI. And especially in the areas where facilities for rapid diagnostic tests are not available.

We constructed a predictive model by multivariate logistic regression analysis based on clinical and laboratory parameters for patients presenting early in the course of the disease before the diagnosis of dengue was established. When the model included only clinical parameters myalgia emerged as a significant predictor of DF. When the model included both clinical and laboratory features, myalgia, leukopenia, and raised AST were significant predictors of DF. This model showed excellent accuracy with good sensitivity, specificity, and predictive values.

Studies involving multiple variables to build a predictive model of dengue by multivariable logistic regression analysis have been reported. ${ }^{4,10,18,21,22,24-27}$
Fernández et al. ${ }^{4}$ observed retro-ocular pain, petechiae and gingival bleeding were associated with an increased risk of DF. Retro-orbital pain was a significant finding in DF in our study but this parameter could not be included for the regression analysis as the number of cases were less. Hence, studies involving a large population are required for the evaluation of this clinical finding.

Daumas et al. ${ }^{18}$ in their predictive model found conjunctival redness and leukocyte count to be associated with dengue while Gregory et al. ${ }^{23}$ observed retro-orbital pain, absence of cough, rash, lack of sore throat and low platelet count as predictors of DF. White blood cell count lower than $5,000 / \mathrm{mm}^{3}$ was the only laboratory parameter that was predictive of DF observed by Chadwick et al. ${ }^{10}$

In a recent study from India differentiating DF from OFI in adults using hematological parameters, WBC, platelets, neutrophils and eosinophils were the parameters predicting DF. ${ }^{25}$ Thrombocytopenia in combination with arthralgia has been reported by Karande et al. ${ }^{26}$

Elevated AST has been reported to be more common among children with DF reaching extremely high levels in certain cases, which reinforces the importance of liver involvement in this disease. Rodrigues et al. observed increased raised AST to be associated with DF. ${ }^{24}$ Potts and Rothman ${ }^{21}$ and Wilder-Smith et al. ${ }^{27}$ also observed elevated AST as an important predictor of dengue in addition to low platelet and WBC count.

Elevated AST with low WBC count and myalgia were reported by Cucunawangsih et al. and Ho et al. ${ }^{28,29}$

We have attempted to build a predictive model consisting of independent parameters that would help to distinguish DF from OFI. The strength of the study was the study period involved two seasons of DF outbreak. This avoided the drawback of clinical and laboratory features that may be specified in a single epidemic season. Also, the model was internally validated.

However, this was a hospital-based study, with a small sample size from a single urban region of Maharashtra State. For a generalization of the results, more studies are required from other parts of the state and this is the limitation of the study.

\section{Conclusion}

The study suggests clinical and laboratory parameters including myalgia, low leukocyte count, and raised AST can be used to identify early dengue infection in children before the laboratory diagnosis is established. It can help the clinicians to make clinical decision, prioritize appropriate management and improve patient outcome.

\section{References}

1. Gubler DJ. The global pandemic of dengue/dengue haemorrhagic fever: current status and prospects for the future. Ann Acad Med Singap 1998;27(2):227-234.

2. Ganeshkumar P, Murhekar MV, Poornima V, et al. Dengue infection in India: a systematic review and meta-analysis. PLoS Negl Trop Dis 2018;12(7):e0006618. DOI: 10.1371/journal.pntd.0006618.

3. Gan VC, Tan L-K, Lye DC, et al. Diagnosing dengue at the point-ofcare: utility of a rapid combined diagnostic kit in Singapore. PLoS One 2014;9(3):e90037. DOI: 10.1371/journal.pone.0090037.

4. Fernández $E$, Smieja M, Walter SD, et al. A predictive model to differentiate dengue from other febrile illness. BMC Infect Dis 2016;16(1):694. DOI: 10.1186/s12879-016-2024-y.

5. Dengue. Guidelines for diagnosis, treatment prevention and control. Geneva, TDR/WHO, 2009.WHO/HTM/NTD/DEN/2009. 
6. Shrivastava A, Dash PK, Tripathi NK, et al. Evaluation of a commercial dengue NS1 enzyme-linked immunosorbent assay for early diagnosis of dengue infection. Indian J Med Microbiol 2011;29(1):51-55. DOI: 10.4103/0255-0857.76525.

7. Stephen S, Charles MP, Anitharaj V, et al. Early dengue diagnosis by nonstructural protein 1 antigen detection: rapid immunochromatography vs two the enzyme-linked immunosorbent assay kits. Indian J Pathol Microbiol 2014;57(1):81-84. DOI: 10.4103/0377-4929.130905.

8. Low JG, Ooi EE, Tolfvenstam T, et al. Early dengue infection and outcome study (EDEN) - study design and preliminary findings. Ann Acad Med Singap 2006;35(11):783-789.

9. Tanner L, Schreiber M, Low JG, et al. Decision tree algorithms predict the diagnosis and outcome of dengue fever in the early phase of illness. PLoS Negl Trop Dis 2008;2(3):e196. DOI: 10.1371/journal. pntd.0000196.

10. ChadwickD, Arch B, Wilder-Smith A, et al. Distinguishing dengue fever from other infections on the basis of simple clinical and laboratory features: application of logistic regression analysis. J Clin Virol 2006;35(2):147-153. DOI: 10.1016/j.jcv.2005.06.002.

11. Siddiqui K. Heuristics for sample size determination in multivariate statistical techniques. World Appl Sci J 2013;27(2):285-287. DOI: 10.5829/idosi.wasj.2013.27.02.889.

12. Bagley $\mathrm{SC}$, White $\mathrm{H}$, Golomb BA. Logistic regression in the medical literature: Standards for use and reporting, with particular attention to one medical domain. J Clin Epidemiol 2001;54(10):979-985. DOI: 10.1016/s0895-4356(01)00372-9.

13. Mishra S, Ramanathan R, Agarwalla SK. Clinical profile of dengue fever in children: a study from Southern Odisha, India. Scientifica (Cairo) 2016;2016:6391594. DOI: 10.1155/2016/6391594.

14. Jain $\mathrm{H}$. Clinical profile and outcome of dengue fever in hospitalized children of South Rajasthan, India. Int J Contemp Pediatr 2016;3(2):546-549. DOI: 10.18203/2349-3291.ijcp20161035.

15. Choudhury J, Mohanty D, Routray SS. Clinical profile and outcome of dengue fever and dengue haemorrhagic fever in pediatric age group. Int J Contemp Pediatr 2016;3(2):442-444. DOI: 10.18203/2349-3291. ijcp20161025.

16. Kumar A, Rongpharpi SR, Duggal SD, et al. Clinical, epidemiological and microbiological profile of dengue fever at a tertiary care Hospital in Delhi, India. J Infect Dis Med 2017;2(2):110. DOI: 10.4172/25761420.1000110.

17. Rose W, Sindhu KN, Abraham AM, et al. Incidence of dengue illness among children in an urban setting in South India: a population based study. Int J Infect Dis 2019;84S:S15-S18. DOI: 10.1016/j.ijid.2019. 01.033.

18. Daumas RP, Passos SRL, Oliveira RVC, et al. Clinical and laboratory features that discriminate dengue from other febrile illnesses: a diagnostic accuracy study in Rio de Janeiro, Brazil. BMC Infect Dis 2013;13:77. DOI: 10.1186/1471-2334-13-77.

19. Premaratna R, Pathmeswaran A, Amarasekara ND, et al. A clinical guide for early detection of dengue fever and timing of investigations to detect patients likely to develop complications. Trans R Soc Trop Med Hyg 2009;103(2):127-131. DOI: 10.1016/j.trstmh.2008.07.024.

20. Deshwal R, Qureshi MI, Singh R. Clinical and laboratory profile of dengue fever. J Assoc Physicians India 2015;63(12):30-32.

21. Potts JA, Rothman AL. Clinical and laboratory features that distinguish dengue from other febrile illnesses in endemic populations. Trop Med Int Health 2008;13(11):1328-1340. DOI: 10.1111/j.1365-3156.2008. 02151.x.

22. Prabhavathi R, Madhusudan SR, Suman MG, et al. Study of clinical and laboratory predictive markers of dengue fever and severe dengue in children. J Pediatr Res 2017;4(6):397-404. DOI: 10.17511/ ijpr.2017.06.08.

23. Gregory CJ, Santiago LM, Argüello DF, et al. Clinical and laboratory features that differentiate dengue from other febrile illnesses in an endemic area--Puerto Rico, 2007-2008. Am J Trop Med Hyg 2010;82(5):922-929. DOI: 10.4269/ajtmh.2010.09-0552.

24. Rodrigues MBP, Freire HBM, Corrêa PRL, et al. Is it possible to identify dengue in children on the basis of Ministry of Health criteria for suspected dengue cases? J Pediatr (Rio J) 2005;81(3):209-215.

25. Priyanka P, Dinesh US. Differentiating between Dengue Fever from Other Febrile Illnesses Using Haematological Parameters. Natl J Lab Med 2018;7(4):PO06-PO10. DOI: 10.7860/NJLM/2018/37424:2311.

26. Karande S, Gandhi D, Kulkarni M, et al. Concurrent outbreak of leptospirosis and dengue in Mumbai, India, 2002. J Trop Pediatr 2005;51(3):174-181. DOI: 10.1093/tropej/fmh100.

27. Wilder-Smith A, Earnest A, Paton NI. Use of simple laboratory features to distinguish the early stage of severe acute respiratory syndrome from dengue fever. Clin Infect Dis 2004;39(12):1818-1823. DOI: $10.1086 / 426029$.

28. Ho TS, Wang SM, Lin YS, et al. Clinical and laboratory predictive markers for acute dengue infection. J Biomed Sci 2013;20(1):75. DOI: 10.1186/1423-0127-20-75.

29. Cucunawangsih C, Dewi BE, Sungono V, et al. Scoring model to predict dengue Infection in the Early Phase of Illness in Primary Health Care Centre. Arch Clin Microbiol 2015;6(4):2. 